\title{
Procedimentos Restauradores e Cuidados com a Saúde Bucal em Indivíduos com Fissura Lábio-Palatina
}

\section{Restorative Procedures and Oral Health Care in Individuals with Cleft Lip and Palate}

\author{
Ricardo Danil Guiraldo*a; Sandrine Bittencourt Bergera; Mateus Rodrigues Tonetto ${ }^{\mathrm{b}}$; Eloisa Aparecida Carlesse Paloco \\ Allan Inácio Ferreira Piauilino ${ }^{\mathrm{b}}$; Arilson Nunes Ferreira de Matos ${ }^{\mathrm{b}}$; Andrei Rosa ${ }^{\mathrm{b}}$; Thais Maria Freire Fernandes ${ }^{\mathrm{a}}$ \\ ${ }^{a}$ Unopar, Programa de Pós-Graduação Stricto Sensu em Odontologia. PR, Brasil. \\ bUniversidade de Cuiabá, Programa de Pós-Graduação Stricto Sensu em Ciências Odontológicas. MT, Brasil. \\ E-mail: rdguiraldo@gmail.com
}

\begin{abstract}
Resumo
Fissura labial e/ou palatina é a forma congênita mais frequente anomalia ocorrendo na região craniofacial, as quais são defeitos orofaciais inatos não sindrômicos, que incluem uma variedade de doenças que afetam a face e a cavidade bucal. O objetivo neste estudo foi realizar um artigo técnico visando esclarecer os procedimentos restauradores e cuidados com a saúde bucal em indivíduos com fissura lábio-palatina. Crianças com fissura lábio-palatina apresentam significativo risco de cárie nos incisivos decíduos, além disso, têm mais superfícies cariadas/restauradas na dentição decídua do que crianças não fissuradas. Assim, deve-se promover o ensinamento de técnicas de higiene bucal para controle desta. Procedimentos restauradores, assim como tratamentos endodônticos são realizados em pacientes com fissura lábio-palatina assim como em pacientes sem fissuras. Para realização dos tratamentos dentários convencionais nas crianças com fissura não tratadas cirurgicamente, o uso de isolamento absoluto tem uma especial importância, pois previne o desconforto causado pelo fluxo constante de água dos instrumentos rotativos e pela presença de resíduos de materiais que podem entrar nas vias aéreas e serem aspirados e/ou deglutidos. Portanto, procedimentos restauradores devem ser realizados em indivíduos com fissura lábio-palatina similarmente a indivíduos que não tenham fissura, entretanto, cuidados tais como isolamento absoluto devem ser tomados e a prevenção é fator fundamental nestes indivíduos.
\end{abstract}

Palavras-chaves: Fenda Labial. Fissura Palatina. Cárie Dentária. Reparação de Restauração Dentária.

\begin{abstract}
Cleft lip and / or palate is the most common congenital anomaly occurring in the craniofacial region, which are non-syndromic inborn orofacial defects, which include a variety of diseases that affect the face and oral cavity. The objective of this study was to carry out a technical article to clarify the restorative procedures and oral health care in individuals with cleft lip and palate. Children with cleft lip and palate have a significant risk of caries in the primary incisors, in addition, they have more decayed/restored surfaces in the primary dentition than noncleft children. Thus, the teaching of oral hygiene techniques to control it should be promoted. Restorative procedures, as well as endodontic treatments are performed in patients with cleft lip and palate as well as in patients without clefts. To perform conventional dental treatments on children with cleft not treated surgically, the use of absolute isolation is of special importance, as it prevents discomfort caused by the constant water flow from rotating instruments and the presence of material residues that may enter the airways and be aspirated and/or swallowed. Therefore, restorative procedures must be performed in individuals with cleft lip and palate similarly to individuals who do not have cleft, however, care such as absolute isolation must be taken, and prevention is a fundamental factor in these individuals.
\end{abstract}

Keywords: Cleft Lip. Cleft Palate. Dental Carie. Dental Cavity. Dental Restoration Repair.

\section{Introdução}

Fendas de lábio e/ou palato são defeitos orofaciais inatos não sindrômicos, que incluem uma variedade de doenças que afetam a face e a cavidade bucal (PALMEIRO et al., 2018). Fissura labial e/ou palatina é a forma congênita mais frequente anomalia ocorrendo na região craniofacial (LEHTONEN et al., 2015). Fendas são divididas em três grupos: Fissura labial; Fissura labial e palatina; ou fissura palatina isolada (LEHTONEN et al., 2015). Fissura labial pode ser unilateral ou bilateral. Alterações na aparência, fala, audição e alimentação podem levar a resultados adversos de longa duração que requerem uma abordagem multidisciplinar para melhorar a função, estética, satisfação, integração social e em geral bem-estar (OOSTERKAMP et al., 2007; GLICK et al., 2017). A proporção de crianças com fissura labial e/ ou palatina, entre 6-36 meses de idade com lesões de cárie dentária, foi relatado ser de 18,9\% (MOURA; ANDRE; LOPEZ, 2013).

De acordo com um estudo anterior (LOCKE; BISHOP, 2011) no qual cirurgiões dentistas deram contribuições consideráveis, enfatizou que uma abordagem de equipe envolvendo muitas especialidades cirúrgicas e odontológicas, incluindo ortodontia, odontopediatria, odontologia restauradora, cirurgia oral e maxilofacial e a terapia da fala e da linguagem era essencial para alcançar ótimos resultados para crianças nascidas com CLP com atendimento multidisciplinar. Além disso, a odontopediatria desempenha uma função fundamental no acompanhamento do crescimento e desenvolvimento craniofacial, na manutenção e promoção da saúde oral para que se possa atingir os padrões de função e estética e dentro da normalidade (RODA; LOPES, 2008). Assim, o objetivo neste estudo foi realizar um artigo técnico 
visando esclarecer os procedimentos restauradores e cuidados com a saúde bucal em indivíduos com fissura lábio-palatina.

\section{Desenvolvimento}

\subsection{Procedimentos restauradores}

Os procedimentos restauradores realizados em pacientes com fissuras lábio-palatina são os mesmos que em pacientes não fissurados (RODA; LOPES, 2008). Com exceção feita à anestesia local do nervo alveolar anterior superior, pois em muitos casos não inerva os incisivos superiores. A disciplina de odontopediatria pode confeccionar mantenedores de espaço e promover o ensinamento de técnicas de higiene bucal para controle destas em crianças. Procedimentos restauradores e tratamentos endodônticos são realizados assim como em pacientes sem fissuras. Para realização dos tratamentos dentários convencionais nas crianças com fissura não tratada cirurgicamente, o uso de isolamento absoluto tem uma especial importância pois previne o desconforto causado pelo fluxo constante de água dos instrumentos rotativos e pela presença de resíduos de materiais que podem entrar nas vias aéreas e serem aspirados e/ou deglutidos (TAGGART, 2009). Aplicação de flúor, controle no irrompimento de dentes decíduos e permanentes e maloclusão são fatores que devem ser avaliados ao longo da vida do paciente. Perante a presença de lesões de cárie, são desaconselhados os procedimentos cirúrgicos, devido ao risco de contaminação da ferida cirúrgica pelos microrganismos. Assim, os procedimentos devem ser realizados anteriormente a procedimentos cirúrgicos.

\subsection{Cuidados com a saúde bucal}

Muitos estudos têm mostrado que as crianças com fissura lábio-palatina estão em uma fase significativa de risco de cárie nos incisivos decíduos, além disso, têm mais superfícies cariadas/restauradas na dentição decídua do que crianças não fissuradas (LAGES; MARCOS; PORDEUS, 2004; BRITTON; WELBURY, 2010). Crianças com fenda palatina podem ser tratadas com uma placa de acrílico infantil préoperatória que é aplicada logo após o nascimento para obturar a fenda palatina. Entretanto, sobre esta placa de acrílico, podese esperar que facilite o início da colonização de Estreptococos mutans e Lactobacilos (BOKHOUT et al., 1996). As leveduras são os fungos mais frequentemente isolados na cavidade bucal e ocorre um aumento na prevalência quando hospedeiro e suas defesas são alteradas por fatores locais. A presença de um material sintético sobrejacente altera o ambiente local por diminuir o fluxo de saliva e diminuir os valores do $\mathrm{pH}$, criando condições que parecem facilitar a aderência desse microrganismo (MATTOS et al., 2009). Portanto, pacientes com fissuras têm uma taxa significativamente maior de colonização oral por Candida albicans em comparação com indivíduos não fissurados, o que varia com a idade, tipo de fenda e o número de intervenções cirúrgicas (RAWASHDEH; AYESH; DARWAZEH, 2011).

\section{Conclusão}

Os procedimentos restauradores devem ser realizados em indivíduos com fissura lábio-palatina similarmente a indivíduos que não tenham fissura, entretanto, cuidados como isolamento absoluto devem ser tomados e a prevenção é fator fundamental nestes indivíduos.

\section{Referências}

BOKHOUT, B. et al. Prevalence of Streptococcus mutans and lactobacilli in 18-month-old children with cleft lip and/or palate. Cleft Palate Craniofac J., v.33, n.5, p. 424-428, 1996. doi: 10.1597/1545-1569_1996_033_0424_posmal_2.3.co_2

BRITTON, K.F.M.; WELBURY, R.R. Dental caries prevalence in children with cleft lip/palate aged between 6 months and 6 years in the West of Scotland. Eur. Arch. Paediatr Dent., v.11, n.5, p.236-241, 2010. doi: 10.1007/BF03262754

GLICK, M. et al. A new definition for oral health supported by FDI opens the door to a universal definition of oral health. Am. J. Orthod. Dentofacia.l Orthop., v.151, n.2, p.229-231, 2017. doi: 10.1016/j.jdent.2016.12.005

LAGES, E.M.B.; MARCOS, B.; PORDEUS, I.A. Oral health of individuals with cleft lip, cleft palate, or both. Cleft Palate Craniofac J., v.41, n.1, p.59-63, 2004. doi: 10.1597/02-058.

LEHTONEN, V. et al. Dental treatment need and dental general anesthetics among preschool-age children with cleft lip and palate in northern Finland. J. Oral Sci., v.123, n.4, p.254-259, 2015. doi: 10.1111/eos.12195.

LOCKE, M.; BISHOP, K. An assessment of the contribution of UK specialists in restorative dentistry to cleft lip and palate services. Br. Dent. J., v.210, n.12, p.E20-E20, 2011. doi: 10.1038/ sj.bdj.2011.142.

MATTOS, B.S. et al. Candida albicans in patients with oronasal communication and obturator prostheses. Braz Dent. J., v.20, n.4, p.336-340, 2009. doi: 10.1590/s0103-64402009000400013.

MOURA, A.M.D.; ANDRE, M.; LOPEZ, M.T. Prevalence of caries in Brazilian children with cleft lip and/or palate, aged 6 to 36 months. Braz Oral Res., v.27, n.4, p.336-341, 2013. doi: 10.1590/s1806-83242013005000009.

OOSTERKAMP, B.C. et al. Satisfaction with treatment outcome in bilateral cleft lip and palate patients. Int J Oral Maxillofac Surg., v.36, n.10, p.890-895, 2007. doi: 10.1016/j.ijom.2007.07.008.

RAWASHDEH, M.A.; AYESH, J.A.; DARWAZEH, A.M. Oral candidal colonization in cleft patients as a function of age, gender, surgery, type of cleft, and oral health. J. Oral Maxillofac. Surg., v.69, n.4, p.1207-1213, 2011. doi: 10.1016/j.joms.2010.02.044.

RODA, S.R.; LOPES, V.L.G.S. Aspectos odontológicos das fendas labiopalatinas e orientações para cuidados básicos. Rev. Ciênc. Méd.., v.17, n.2, p.95-103, 2008.

TAGGART, J.C. Handbook of Dental care: diagnostic, preventive and restorative services. New York: Nova Science Publishers, 2009. 